EDUCATION

Research, lmovoction ond Solutions on-line
PSYCHOLOGY

I+D+i
Electronic Journal of Research

in Educational Psychology

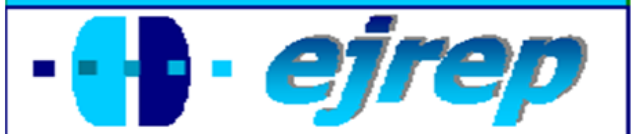

\title{
Relación entre el apoyo social, la satisfacción vital y las expectativas de futuro de menores acogidos en centros de protección
}

\author{
Juan Manuel Fernández Millán ${ }^{1}$, \\ Desirée Díez de la Cortina ${ }^{2}, \mathbf{M}^{\mathrm{a}}$ José Malpica Buitrago $^{2}$, \\ Andrés Hamido Mohamed ${ }^{2}$
}

${ }^{1}$ Dpto. de Psicología Social, Universidad de Granada, Melilla

${ }^{2}$ Centro Asistencial de Melilla

\section{España}

Correspondencia: Juan Manuel Fernández Millán. Poeta Salvador Rueda, 11, $3^{\circ}$ A. 52006 Melilla. España. E-mail: s4527261@ugr.es

(C) Education \& Psychology $\mathrm{I}+\mathrm{D}+\mathrm{i}$ and Editorial EOS (Spain) 


\section{Resumen}

Introducción. El presente trabajo muestra la relación entre las variables percepción del apoyo social, satisfacción con la vida y expectativas de futuro, variables que tienen gran relevancia sobre la adaptación social y personal de los menores.

Método. La muestra esta compuesta por 200 menores, divididos en dos grupos: menores residentes en centros de acogida y menores que viven con sus familias. Ambos grupos han sido evaluados utilizando la Escala de Satisfacción con la Vida

Resultados. Los resultados muestran una correlación negativa entre la situación de institucionalización y la percepción de apoyo familiar, así como, correlaciones positivas entre esta percepción y la satisfacción con la vida y las expectativas de futuro

Discusión y Conclusiones. De los datos obtenidos puede concluirse la importancia de desarrollar redes de apoyo social para mejorar la satisfacción con la vida actual y futura

Palabras Clave: Apoyo social, satisfacción con la vida, expectativas, acogimiento residencial.

Recibido: 24/11/09 Aceptación Inicial: 30/01/10 Aceptación Definitiva: 19/04/10 


\title{
The relationship between social support, satisfaction with life and expectations for the future in minors living in shelter care centres
}

\begin{abstract}
Introduction. The present work shows the relation between the variables perception of the social support, satisfaction with the life and expectations of future, variables that have great relevancy on the social and personal adjustment of the minors.
\end{abstract}

Method. The sample was composed by 200 minors, divided in two groups: resident minors in centers of reception and those who lived with their families. Both groups have been evaluated using the Satisfaction with Life Scale.

Results. The results show a negative correlation between the situation of institutionalization and the perception of familiar support, as well as, positive correlations between this perception and the satisfaction with the life and the expectations of future

Discussion and Conclusions. Of the obtained information one can conclude the importance of developing networks of social support to improve the satisfaction with the current and future life

Keywords: Social support network, satisfaction with life, expectations, residential child care.

Recibido: 11/24/09 Aceptación Inicial: 01/30/10 Aceptación Definitiva: 04/19/10 


\section{Introducción}

Entre los principales recursos de los que dispone el adolescente, se encuentra el apoyo percibido de sus redes sociales, entre las que destaca la familia y el grupo de iguales, que aportan apoyo social a través de frecuentes interacciones, tanto individuales como grupales (Demaray y Malecki, 2002; Levitt, Guacci-Franco y Levitt, 1993). En este sentido Scandroglio, López Martínez y San Jose Sebastián (2008) consideran que en el grupo de iguales y gracias a éste se resuelven algunos de los cometidos más cruciales del desarrollo humano: construir y ensayar su identidad personal y de género, desarrollar habilidades y competencias sociales así como defineir un sistema de valores y creencias.

Se define apoyo social como un conjunto de provisiones expresivas o instrumentales que nos aporta nuestra comunidad y medio social, tanto en situaciones habituales como en las inusuales (Lin y Ensel, 1989), desempeñando funciones emocionales y materiales. Disponer de personas de confianza a las que poder expresar nuestras emociones, problemas o dificultades, de las que escuchar su opinión, o simplemente tener la sensación de que somos escuchados y aceptados como personas, ha demostrado tener un efecto positivo tanto en la autoestima como en la capacidad para afrontar de forma adecuada las situaciones difíciles y estresantes (Cava, 1995; Herrero, 1994; Lin y Ensel, 1989; Musitu, Buelga, Lila y Cava, 2001).

La relación del apoyo social con la adaptación social y personal de los menores (niños $\mathrm{y}$ adolescentes) ha sido estudiado en numerosos trabajos (Armstrong, Birnie-Lefcovitch y Ungar, 2005; Barra, 2004; Galván et al., 2006; García y Herrero, 2006; Martin y Dávila, 2008; Méndez y Barra, 2008; Vranceanu, Hobfoll y Johnson, 2007). Bravo y Fernández, (2003) compararon un grupo de adolescentes acogidos en hogares de protección con otro que vivían con su familia concluyendo que los menores acogidos percibían menor apoyo social que los menores que vivían en su hogar familiar. Más recientemente, Martin y Dávila (2008), han realizado una investigación en la que la muestra estaba formada por menores residentes en centros de protección, encontraron que el apoyo familiar no presentaba relación con la adaptación de los menores, mientras que aparecía una marcada incidencia sobre la adaptación y el apoyo recibido por adultos extrafamiliares (profesores, educadores, entrenadores o monitores de ocio). Méndez y Barra (2008) han estudiado la percepción del apoyo social entre adolescentes infractores y no infractores pareados en diversas variables sociodemográficas 
encontrando diferencias significativas entre distintas dimensiones (emocional, consejo, instrumental y reciprocidad) que componen el apoyo. Recientemente, Martínez-González, Inglés, Piqueras y Ramos (2010) han realizado una revisión sobre el papel clave de los amigos y los padres en el bienestar emocional y físico, además de en el rendimiento académico, detallando las consecuencias de los distintos estilos educativos (habilidades educativas) en la formación de la personalidad y el autocontrol del joven.

El apoyo social se relaciona entre otras variables con la satisfacción con la vida, componente cognitivo del constructo "bienestar subjetivo" que se ha definido como un sentimiento de bienestar consigo mismo, que algunos autores han insistido en la necesidad de diferenciarlo de la aprobación-desaprobación o del grado de satisfacción con las condiciones objetivas de vida; siendo básicamente una estimación subjetiva (Undurraga y Avendaño, 1998). Los individuos que informan una alta satisfacción con su propia vida gozarían de una apropiada salud mental, ausente de afectos negativos, ansiedad, estrés o depresión (Atienza, Pons, Balaguer y García-Merita, 2000).

Por último, las expectativas de futuro son entendidas, en este estudio, como la perspectiva que tiene el sujeto sobre la calidad de vida (en sentido amplio) que cree que alcanzará. De la propia definición de satisfacción en la vida, se desprende que cualquier variable que altere o defina los distintos dominios implicados en la valoración cognitiva de la propia vida, afectará a la propia percepción de satisfacción de vida. En el caso que nos ocupa, la variable que hemos tenido en cuenta es el grado de apoyo familiar. Para ello hemos utilizado dos grupos de menores que contarían con un apoyo social diferenciado: Menores que viven con sus familias en un hogar propio y menores en desamparo residentes en centros de acogida. Partimos del supuesto de que "si estos menores han tenido que llegar a vivir en un hogar de protección, es precisamente porque su familia no provee protección ni apoyo social” (Martin y Dávila, 2008: 229).

En el presente trabajo se estudia la relación existente entre 3 variables cognitivas, la percepción del apoyo social, la satisfacción con la vida y las expectativas de futuro en menores adolescentes y preadolescentes, acogidos en centros de protección en comparación con otro grupo de menores que vive con su familia. La presente investigación tiene como objetivos comprobar si los menores acogidos perciben un menor apoyo familiar. Otro de los objeti- 
vos será verificar la relación entre la satisfacción con la vida y la percepción del apoyo social-familiar. Por último, se pretende evidenciar la relación entre el apoyo familiar y las expectativas de futuro.

\section{Método}

\section{Participantes}

La muestra del estudio se compone de 200 sujetos de los que se han descartado 21 por diferentes motivos de carácter metodológicos ${ }^{1}$. La muestra definitiva $(\mathrm{N}=179)$ comprende un rango de edad de 11 a 18 años de los que el 50,3 \% son chicas y el 49,7 \% son chicos. Respecto al grupo al que pertenecen, el $57 \%$ no se encuentra institucionalizado ${ }^{2}$, mientras que el $43 \%$ sí lo está (tabla 1 y 2 ).

Tabla 1. Datos descriptivos (frecuencias y porcentajes) de la edad de los sujetos de las distintas muestras.

\begin{tabular}{rrrrrrr}
\hline & \multicolumn{2}{c}{ Muestra total } & \multicolumn{2}{c}{ Menores en familia } & \multicolumn{2}{c}{ Menores acogidos } \\
\hline Edad & Frecuencia & Porcentaje & Frecuencia & Porcentaje & Frecuencia & Porcentaje \\
\hline 11,00 & 3 & 1,7 & 0 & 0 & 3 & 3,9 \\
12,00 & 11 & 6,1 & 2 & 2,0 & 9 & 11,7 \\
13,00 & 20 & 11,2 & 13 & 12,7 & 7 & 9,1 \\
14,00 & 35 & 19,6 & 17 & 16,7 & 18 & 23,4 \\
15,00 & 32 & 17,9 & 21 & 20,6 & 11 & 14,3 \\
16,00 & 25 & 14,0 & 13 & 12,7 & 12 & 15,6 \\
17,00 & 42 & 23,5 & 25 & 24,5 & 17 & 22,1 \\
18,00 & 11 & 6,1 & 11 & 10,8 & 0 & 0 \\
Total & 179 & 100,0 & 102 & 100,0 & 77 & 100,0 \\
\hline
\end{tabular}

\footnotetext{
${ }^{1}$ Contestación incorrecta de los cuestionarios o falta de datos en los mismos.

${ }^{2}$ Cuando hablamos de institucionalización, nos referimos a menores que, por distintas causas (situación de desamparo), han sido acogidos en centros de menores.
} 
Tabla 2. Datos descriptivos (frecuencias y porcentajes) de la distribución por sexo de los sujetos de las distintas muestras

\begin{tabular}{crrrrrr}
\hline & \multicolumn{2}{c}{ Muestra total } & \multicolumn{2}{c}{ Menores en familia } & \multicolumn{2}{c}{ Menores acogidos } \\
\hline Sexo & Frecuencia & Porcentaje & Frecuencia & Porcentaje & Frecuencia & Porcentaje \\
\hline Chicas & 90 & 50,3 & 59 & 57,8 & 31 & 40,3 \\
Chicos & 89 & 49,7 & 43 & 42,2 & 46 & 59,7 \\
Total & 179 & 100,0 & 102 & 100,0 & 77 & 100,0 \\
\hline
\end{tabular}

\section{Instrumento}

El instrumento de evaluación utilizado es la Escala de Satisfacción con la Vida- The Satisfacction With Life Scale- (Diener, Emmons, Larsen y Griffin, 1985), adaptación al castellano de Atienza et al. (2000). Este cuestionario consta de 5 ítems que se contestan sobre una escala tipo Likert de 1 a 4 en función del grado de acuerdo o desacuerdo (véanse los ítems en el anexo). El instrumento mostró buenas propiedades psicométricas con un alfa de Cronbach de 0,84 en la adaptación al castellano.Junto a este instrumento se pasó un cuestionario con 2 ítems, uno de ellos con la intención de medir el apoyo por parte de la familia (escala de 6 puntos) y el otro para registrar las expectativas de vida (escala de 5 posibilidades -muy mala, mala, normal, buena, muy buena-).

\section{Procedimiento}

La evaluación de los sujetos pertenecientes a hogares familiares fue realizada en diferentes Institutos de Educación Secundaria de Melilla y de la provincia de Málaga durante el horario escolar. Previamente, se le proporcionaron instrucciones para contestar al cuestionario que se rellenaron en presencia de un entrevistador. A los sujetos acogidos en centros (de la ciudad de Melilla), se les evaluó dentro de sus respectivas instituciones, de forma individual. Estas instituciones dependen de la Consejería de Bienestar Social y su capacidad varía entre 8 y 25 menores acogidos.

\section{Diseño}

Siguiendo la clasificación de Montero y León (2007), este trabajo correspondería a un diseño de Estudio ex post-facto retrospectivo de dos grupos, uno de cuasi control. 


\section{Resultados}

Las correlaciones encontradas entre la institucionalización y el apoyo familiar, muestran que la institucionalización covaría de forma negativa y significativamente con el apoyo familiar $(\mathrm{r}=-0,284 ; \mathrm{p}<0,01)$ (Tabla 3). La prueba no paramétrica de Mann-Whitney (Tabla 4) muestra una significación asintótica de 0,001 por lo que puede afirmarse que los niños y niñas institucionalizados perciben menor apoyo familiar que los menores que viven en sus hogares familiares.

Tabla 3. Correlaciones entre distintas variables cognitivas y estar acogido en un centro (institucionalización)

\begin{tabular}{lc}
\hline & \multicolumn{2}{c}{ INSTITUCIONALIZACION } \\
\hline satisfacción &,$- 376(* *)$ \\
apoyo &,$- 284(* *)$ \\
expectativas &,- 051 \\
\hline$*$ La correlación es significante al nivel 0,05 (bilateral). \\
$* *$ La correlación es significativa al nivel 0,01 (bilateral).
\end{tabular}

Tabla 4. Prueba Mann-Whitney para las variables satisfación, apoyo social y expectativas de futuro tomando como variable de agrupación el acogimiento en centros (institucionalización)

\begin{tabular}{lccc}
\hline & satisfacción & apoyo & expectativas \\
\hline U de Mann-Whitney & 2180,500 & 2831,000 & 3727,000 \\
W de Wilcoxon & 5183,500 & 5834,000 & 6730,000 \\
Z & $-5,109$ & $-3,361$ &,- 609 \\
Sig. asintót. (bilateral) &, 000 &, 001 &, 542 \\
\hline a Variable de agrupación: INSTITUCIONALIZACION
\end{tabular}

También puede observarse que el apoyo familiar aumenta la percepción de satisfacción $(\mathrm{r}=0.380 ; \mathrm{p}<0.01)$ (Tabla 5). La prueba no paramétrica de Mann-Whitney muestra una significación asintótica de 0.000 lo que indica diferencias significativas en la satisfacción con la vida entre los menores institucionalizados y los que no lo están. Apareciendo los menores institucionalizados con puntuaciones que reflejan menor satisfacción con la vida (Tabla 4). 
Por último, la correlación entre apoyo familiar y expectativas es positiva y significativas $(r=0.459 ; p<0.001)$ (Tabla 4), lo que indica que los menores que perciben mayor apoyo, muestran mejores expectativas de futuro. Sin embargo, la prueba no paramétrica de comparación de medias entre institucionalización y expectativas no es significativa (0.542) poniendo de manifiesto que el hecho de estar institucionalizado no implica peores expectativas respecto al futuro. Resultado que se discutirá más adelante.

Tabla 5.Correlaciones entre las variables satisfacción con la vida, apoyo social y expectativas de futuro.

\begin{tabular}{lccc}
\hline & satisfacción & apoyo & expectativas \\
\hline satisfacción & 1 &, $380(* *)$ &, $412(* *)$ \\
apoyo &, $380(* *)$ & 1 &, $459(* *)$ \\
expectativas &, $412(* *)$ &, $459(* *)$ & 1 \\
\hline * La correlación es significante al nivel 0,05 (bilateral). \\
$* *$ La correlación es significativa al nivel 0,01 (bilateral).
\end{tabular}

\section{Discusión y conclusiones}

Los resultados que se han obtenido en este estudio confirman la idea de que el apoyo familiar es una variable estrechamente unida a la satisfacción y a las expectativas percibidas, coincidiendo estos datos con los hallados por Díaz y Sánchez-López (2002).

La falta de relación hallada entre apoyo y expectativas entre los menores acogidos puede deberse a que el menor institucionalizado compensaría el menor apoyo familiar con la mejora en otros dominios (amistad, tiempo libre, salud) lo que se traduce en una compensación en sus expectativas (Abbey y Andrews, 1985). Esta hipótesis podría comprobarse en futuros estudios en los que se tengan en cuenta variables más específicas como la edad de los menores, el tiempo de institucionalización la frecuencia de visitas de los padres, así como los cambios sufridos desde su institucionalización a nivel de amistades, dedicación del tiempo libre o salud. 
Como se ha anotado anteriormente, los niños acogidos en centros presentan un nivel de insatisfacción mayor que aquellos que viven con sus familias. Este dato puede explicarse por el hecho de que, por un lado, como apuntan algunos autores (Casas et al., 2004; Huebner, 1991) existe relación entre la autoestima y la satisfacción por la vida y, por otra parte que, las puntuaciones en autoestima de los menores en desamparo corresponden a percentiles bajos.

Concluyendo, se puede afirmar que los menores acogidos perciben menor apoyo familiar y que esta percepción se relaciona con la satisfacción con la propia vida y con las expectativas que el menor se hace sobre su futuro. Estos hallazgos deben tenerse como base para incluir acciones dirigidas al acercamiento y al apoyo parental con los familiares de los menores acogidos mientras perdure la estancia de éstos en el centro.

\section{Referencias}

Abbey, A. y Andrews, F. (1985). Modeling the psychological determinants of life quality. Social Indicators Research, 16, 1-34.

Armstrong, M., Birnie-Lefcovitch, S. y Ungar, M. T. (2005). Pathways Between Social Support, Family Well Being, Quality of Parenting, and Child Resilience: What We Know. Journal of Child and Family Studies, 14(2), 269-281.

Atienza, F. L., Pons, D., Balaguer, I. y García-Merita, M. (2000). Propiedades psicométricas de la escala de satisfacción con la vida en adolescentes. Psicothema, 12(2), 314-320. .

Barra, E. (2004). Apoyo social, estrés y salud. Psicología y Salud, 14, 237-243.

Bravo, A. y Fernández, J. (2003). Las redes de apoyo social de los adolescentes acogidos a residencias de protección. Un análisis comparativo con población normativa. Psicothema, 15 , 136-142.

Casas, F., Buxarrais, M., Figuer, C., González, M., Tey, A., Noguera, E. y Rodríguez, J.M. (2004). Los valores y su influencia en la satisfacción vital de los adolescentes entre los 12 y los 16 años: estudio de algunos correlatos. Apuntes de psicología, 22 , 3-23.

Cava, M. (1995). Autoestima y apoyo social: Su incidencia en el ánimo depresivo en unamuestra de jóvenes adultos universitarios. Tesis doctoral no publicada. Universitat deValència, Valencia. 
Demaray, M. y Malecki, C. (2002). The relationship between perceived social support and maladjustment for students at risk. Psychology in the Schools, 39(3), 305 - 316.

Díaz, J. y Sánchez-López, M. (2002). Relaciones entre estilos de personalidad y satisfacción autopercibida en diferentes áreas vitales. Psicothema, 14(1), 100-105.

Diener, E., Emmons, R., Larsen, R. y Griffin, S. (1985). The Satisfaction With Life Scale. Journal of Personalitiy Assessment, 49 , 71-75.

Galván, J., Romero, M., Rodríguez, E., Durand, A., Colmenares, E. y Saldivar, G. (2006). La importancia del apoyo social para el bienestar físico y mental de las mujeres reclusas. Salud Mental, 29 , 68-74.

García, E. y Herrero, J. (2006). La comunidad como fuente de apoyo social. Evaluación e implicaciones en los ámbitos individual y comunitario. Revista Latinoamericana de Psicología, 38, 327-342.

Herrero, J. (1994). Estresores sociales y recursos sociales: El papel del apoyo social en el ajuste bio-psico-social. Tesis doctoral no publicada. Universitat de València, Valencia

Huebner, E. (1991). Initial Development of the Student's Life Satisfaction Scale. Social Psychology International, 12, 231-240.

Levitt, M., Guacci-Franco, N. y Levitt, J. (1993). Convoys of social support in childhood and earlyadolescence: structure and function. Developmental Psychology, 29(5), 811-818.

Lin, N. y Ensel, W. (1989). Life stress and health: Stressors and resources. American Sociological Review, 54, 382-399.

Martin, E. y Dávila, L. (2008). Redes de apoyo social y adaptación de los menores en acogimiento residencial. Psicothema, 20(2), 229-235.

Martínez-González, A. E., Inglés, C. J., Piqueras, J. A. y Ramos, V. (2010). La importancia de los amigos y los padres en la salud y el rendimiento escolar. Electronic Journal of Reserch in Educational Psychology, 8(1), 111-138.

Méndez, P. y Barra, E. (2008). Apoyo social percibido en adolescentes infractores de Ley y no infractores. Psykhe, 17(1), 59-64.

Montero, I. y Leó, O.G. (2007). A guide for naming research studies in Psychology. International Journal of Clinical and Health Psychology, 7 (3), 847-862

Musitu, G., Buelga, S., Lila, M. y Cava, M. (2001). Familia y adolescencia: Análisis de un modelo de intervención psicosocial. Madrid: Síntesis. 
Scandroglio, B., López Martínez, J., San Jose Sebastián, M. (2008). “Pandillas”: grupos juveniles y conductas desviadas. La perspectiva psicosocial en el análisis y la intervención. Electronic Journal of Research in Educational Psychology, 14, 6 (1), 65-94.

Undurraga, C. y Avendaño, C. (1998). Dimensión psicológica de la pobreza. Psykhe, 6(1), 5763.

Vranceanu, A., Hobfoll, S. y Johnson, R. (2007). Child multi-type maltreatment and associated depression and PTSD symptoms: the role of social support and stress. Child Abuse Neglect, 31(1), 71-84.

Anexo. Ítems que componen la Escala de Satisfacción con la Vida-The Satisfacction With Life Scale- (Diener, Emmons, Larsen y Griffin, 1985)

1. Mi vida es en la mayoría de los aspectos como me gustaría que fuera $\begin{array}{llll}1 & 2 & 3 & 4\end{array}$

2. Hasta ahora, todo lo que me gustaría tener en mi vida ya lo tengo. $\begin{array}{llll}1 & 2 & 3 & 4\end{array}$

3. No estoy contento/a con mi vida.

4. Si pudiera vivir mi vida otra vez, me gustaría que fuera como ha sido hasta ahora-- 